\title{
Evolution of COVID-19 management in critical care: review and perspective from a hospital in the United Kingdom
}

\author{
Avinash Kumar Jha, Sudhindra Gurunath Kulkarni \\ Critical Care Unit, Royal Preston Hospital, Preston, UK
}

The unexpected emergence and spread of coronavirus disease 2019 (COVID-19) has been pandemic, with long-lasting effects, and unfortunately, it does not seem to have ended. Integrating advanced planning, strong teamwork, and clinical management have been both essential and rewarding during this time. Understanding the new concepts of this novel disease and accommodating them into clinical practice is an ongoing process, ultimately leading to advanced and highly specific treatment modalities. We conducted a literature review through PubMed, Europe PMC, Scopus, and Google Scholar to incorporate the most updated therapeutic principles. This article provides a concise and panoramic view of the cohort of critically ill patients admitted to the intensive care unit. We conclude that COVID-19 management includes low tidal volume ventilation, early proning, steroids, and a high suspicion for secondary bacterial/fungal infections. Lung ultrasound is emerging as a promising tool in assessing the clinical response. Managing non-clinical factors such as staff burnout, communication/ consent issues, and socio-emotional well-being is equally important.

Key Words: burnout; COVID-19; critical care; delirium; hydroxychloroquine

\section{INTRODUCTION}

COVID-19 has been one of the most devastating and debilitating diseases experienced in recent decades. As of January 2021, 3.3 million have tested positive, and 89,000 deaths have been reported in the United Kingdom. The second wave has wreaked havoc by placing healthcare professionals and resources into tremendous pressures. With the advent of electronic media, much literature has been made available in rapid succession leading to an information explosion: filtering through these vast resources and their application in clinical practice is a difficult task. Therefore, we have aimed to simplify, discuss, and summarise the evidence base and the management of COVID-19 patients in the intensive care unit (ICU) using a pragmatic and holistic approach, covering clinical and non-clinical aspects.

\section{BEGINNING, PREPARATION, AND MANAGEMENT}

In the initial days of January 2020, it was evident that borders will not restrict COVID-19; a few weeks later it had made its appearance in Europe. At this time, our unit started formulating

\section{Review Article}

Received: October 21, 2020

Revised: January 24, 2021

Accepted: January 25, 2021

Corresponding author Avinash Kumar Jha Critical Care Unit, Royal Preston Hospital, Sharoe green lane, Fulwood, Preston PR2 9WL, UK Tel: +44-7828942895

E-mail: avinash.jha@Ithtr.nhs.uk

Copyright (@) 2021 The Korean Society of Critical Care Medicine

This is an Open Access article distributed under the terms of Creative Attributions Non-Commercial License (https:// creativecommons.org/li-censes/by-nc/4.0/) which permits unrestricted noncommercial use, distribution, and reproduction in any medium, provided the original work is properly cited. 
plans and protocols. Promptly, we were all fitted with masks and attended simulations on the use of personal protective equipment (PPE) and intubation drills, a team of members assessed the staff for risk, and triage pathways were finalised. Separate dedicated areas were pre-allocated for suspected cases awaiting COVID-19 results, or computed tomography (CT) scans and for confirmed cases of COVID-19 (i.e., positive swabs/CT findings).

We revisited the process of transfer to CT scan console. A separate corridor created specifically for suspected/confirmed COVID-19 with alerts and signposts. We used a dedicated scanner for COVID-19 to limit the spread of infection to other patients and all staff members involved in the scan donned full PPE. Since there is a network of ICUs at regional and national levels, there was close coordination and communication regarding any necessary modifications. It was ensured that all centres followed a unified system regarding the management and reporting of cases.

Being an unprecedented infection, clinical management of these cases was not straightforward. As expected, it took a moderate amount of time for all the health care workers to get beyond an acceptable point on the learning curve. Adapting to PPE for long hours, new clinical protocols, a unique style of ward rounds, and increased reliance on airway-trained personnel were some of the newfound changes to our daily routine on the unit. The intensive care received admissions from wards, and there were dedicated isolated wards for suspected cases and confirmed cases with less severe illness. We quickly adapted to self-proning in awake patients as per the Intensive Care Society (ICS) [1]; A study performed on 50 patients in the emergency department showed improved oxygenation on awake self proning [2]. We also observed the associated improved oxygenation and are awaiting more significant trials for the evidence base underlying this. Even though these patients did not complain of respiratory distress, their oxygenation was poor. The mechanism was explained by breathing larger tidal volumes to compensate for increased minute ventilation instead of increased respiratory rate.

Furthermore, Gattinoni et al. [3] have mentioned patient self-inflicted lung injury to explain the deranged pulmonary function. We utilised early transfer to ICU of these cases if there were objective signs of worsening, namely increased work of breathing, oxygen requirement greater than $50 \%$, or respiratory rate greater than 30 ; these were essentially the components that would guide us in evaluating increased work of breathing in conjunction with clinical assessment. Our admission protocol to critical care unit is shown in Figure 1.

\section{KEY MESSAGES}

- Timely planning and resource allocation: this is of vital importance, having management protocols can minimise deviations from standard care and lead to better patient outcomes.

- Staff burnout and well-being: these play a significant role in the effectiveness of teamwork and can influence patient outcomes.

- Delirium management: this needs a multidisciplinary team approach.

\section{Airway Management}

On ICU admission, helmet continuous positive airway pressure (CPAP) was the initial mode of ventilation, which is in keeping with the evidence, as suggested by the ICS [4]. Measurements that require advanced monitoring such as oesophageal pressures and airway occlusion pressure (P0.1) are objective markers of work of breathing. Still, as these are cumbersome and not commonly available, their practical application has been inconvenient. Zhou et al. [5], in their study, mentioned mechanically ventilated patients having a mortality of 97\%, whereas Barrasa et al. [6] reported relatively lower mortality, which they attributed to early intubation, use of the highflow nasal cannula (HFNC) and avoidance of CPAP. In terms of CPAP failure, a persistent requirement of fractional inspiratory oxygen levels of greater than $70 \%$, respiratory rate of more than 30 for 48 hours suggested inadequate response to CPAP. We use these variables along with the clinical picture to guide us in decision making towards endotracheal intubation. However, few patients on our unit required early or immediate intubation based on the insignificant changes in oxygenation despite CPAP therapy.

There has been a suggestion favouring HFNC instead of CPAP [7], albeit this increases the aerosol generation/transmission, and we do not use this. At this point, it is not clear which of these ventilatory modalities will be of most benefit. The Recovery-RS trial is underway; hopefully, it will give better insight. Our airway management protocols regarding checklists and difficult intubations remained the same across all specialities, including anaesthetics, the emergency department (ED), and ICU, as depicted in (Figures 2 and 3) [8] .

\section{Phenotypes}

A new concept has classified the patients into phenotypes L and $\mathrm{H}$. The $\mathrm{L}$ type receives liberal tidal volumes and positive end-expiratory pressure (PEEP) of less than $10 \mathrm{~cm} \mathrm{H}_{2} \mathrm{O}$. The $\mathrm{H}$ 

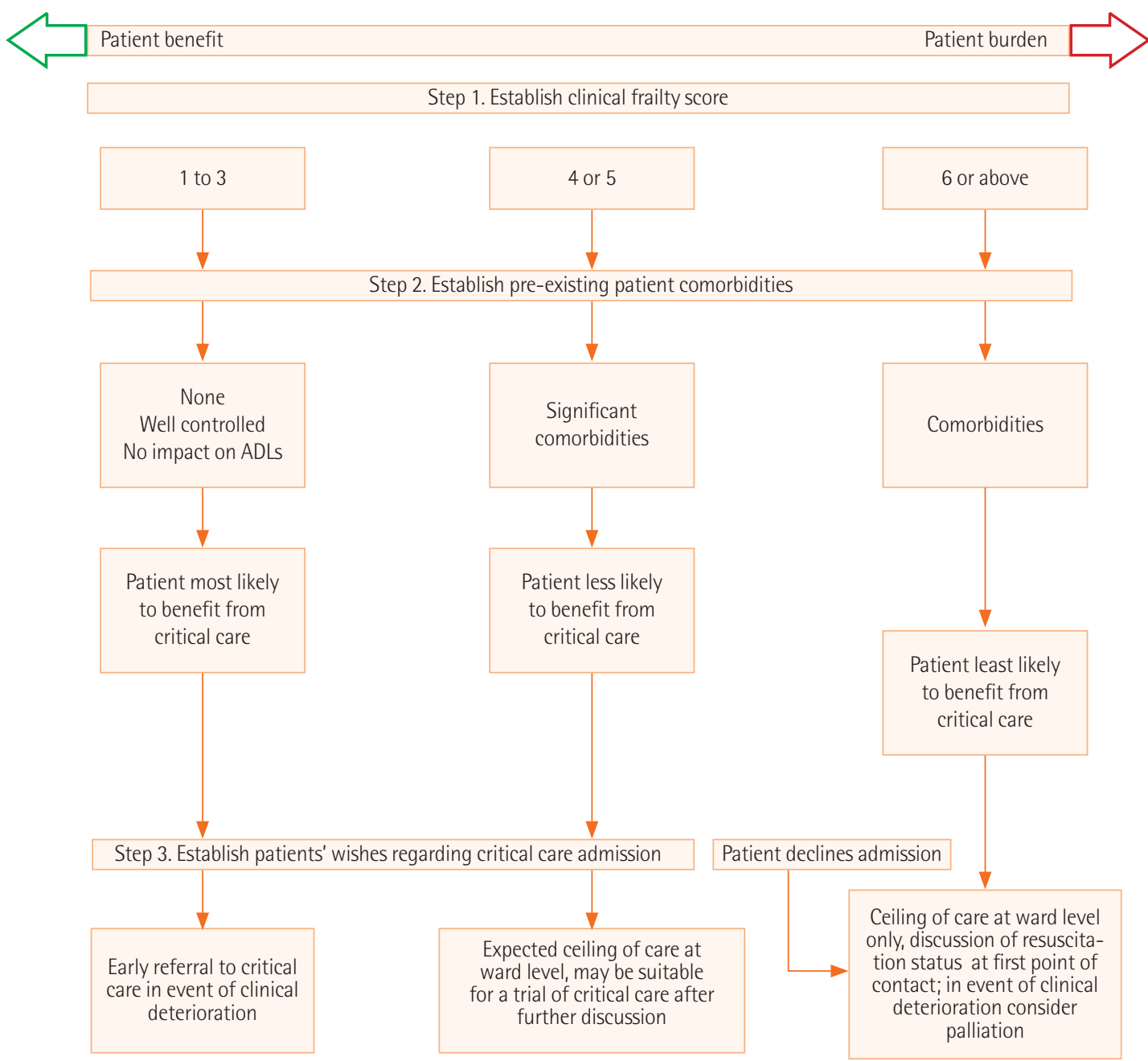

Figure 1. Royal Preston Hospital critical care admission protocol. ADL: activities of daily living.

type management is on the same lines as for acute respiratory distress syndrome (ARDS). This classification is based mainly on the chest CT findings; parameters like compliance, elastance, and lung weight are less sensitive in differentiating between the two groups. It is also evident that patients transition from $\mathrm{L}$ type to $\mathrm{H}$ type [3]. As per our observation, this progression could either represent different stages on the spectrum of the same disease or be secondary to ventilator-induced lung injury. Furthermore, this classification extended into a total of five phenotypes based on their features, of which type 5 is present in cases that were on helmet CPAP before intubation and had elevated procalcitonin [9]. The CT scan findings of the phenotypes are evident in Figure 4A.

Interestingly, a Spanish multi-center trial found similar physiologic variables: driving pressure, respiratory system compliance and plateau pressure between COVID and non-COVID ARDS [10].
Even before COVID-19, Calfee et al. [11] identified subtypes in ARDS as hypoinflammatory and hyperinflammatory, with the latter associated with a worse prognosis. A correlation between the subtypes and phenotypes needs evaluation in future studies. Despite the countertheory that COVID-19 management should be on ARDS lines, our ventilation strategy is individualized based on phenotype classification awaiting further evidence about the optimal approach.

\section{Imaging}

There was a clear transition towards more high-resolution CT (HRCT) chest and CT pulmonary angiography (CTPA) as the days progressed. HRCT was used to confirm diagnosis given its higher sensitivity compared to Reverse transcriptase-polymerase chain reaction [12]. We could simultaneously understand the phenotype and manage it accordingly. Adding a CTPA in the same session helped us diagnose pulmonary em- 


\begin{tabular}{|c|c|c|c|c|}
\hline Equipment check & Airway plan & PPE & In COVID area & After intubation \\
\hline $\begin{array}{l}\checkmark \text { Allocate roles } \\
\text { - Team leader } \\
\text { - Intubator } \\
\text { - Cricoid performer } \\
\text { - Airway assistant } \\
\text { - Drugs injector } \\
\text { - Runner } \\
\checkmark \text { Any allergies? } \\
\checkmark \text { Weight? }\end{array}$ & \multirow{4}{*}{$\begin{array}{l}\checkmark \text { Anticipated difficult airway? } \\
\checkmark \text { Difficult airway trolley } \\
\checkmark \text { Video laryngoscope available? } \\
\checkmark \text { Second intubator available? } \\
\checkmark \text { Discuss plan } \\
\text { - Plan A: RSI } \\
\text { - Plan B/C: BMV by } 2 \text { hands } \\
2 \text { people } \& \text { SGA } \\
\text { - Pland D: scalpel cricothyroid- } \\
\text { otomy (FONA) } \\
\text { - Who will perform FONA? } \\
\checkmark \text { Plan approved? } \\
\checkmark \text { Any concerns? }\end{array}$} & \multirow[t]{4}{*}{$\begin{array}{l}\checkmark \text { Hand hygiene } \\
\checkmark \text { Donning PPE } \\
\checkmark \text { Mask/respirator } \\
\checkmark \text { Visor } \\
\checkmark \text { Gloves } \\
\checkmark \text { Name/role labels on PPE } \\
\checkmark \text { Final PPE check by colleague }\end{array}$} & \multirow{4}{*}{$\begin{array}{l}\checkmark \text { Confirm patient } \\
\checkmark \text { Apply monitors } \\
-\mathrm{SpO}_{2}, \mathrm{BP}, \mathrm{ECG}, \mathrm{EtCO}_{2} \\
\checkmark \text { Assess airway } \\
- \text { MACOCHA score } \\
\text { - Locate cricothyroid mem- } \\
\quad \text { brane } \\
\checkmark \text { NG aspirated? } \\
\checkmark \text { Preoxygenation } \\
\text { - Avoid NIV/HFNC } \\
\checkmark \text { Runner on walkie-talkie/ } \\
\quad \text { wireless phone } \\
\checkmark \text { Intravenous access } \times 2 \\
\checkmark \text { IV fluid running? } \\
\checkmark \text { Patient position optimized? }\end{array}$} & \multirow{2}{*}{$\begin{array}{l}\checkmark \text { In covid area } \\
\text { - Confirm EtCO2 reading } \\
\text { - Inflate cuff } \\
\text { - Secure ET tube } \\
\text { - Ventilator on standby during } \\
\text { disconnections } \\
\text { - Good seal at connections } \\
\checkmark \text { Nasogastric tube (if needed) } \\
\checkmark \text { Chart intubation grade at } \\
\text { bedside } \\
\checkmark \text { Safe disposal of equipment/ } \\
\text { sharps }\end{array}$} \\
\hline $\begin{array}{l}\checkmark \text { Intubation grab kit } \\
\text { - Suction } \\
\text { - Naso/oropharyngeal airway } \\
\text { - Bains/Mapleson C+HME } \\
\text { - } \mathrm{EtCO}_{2}\end{array}$ & & & & \\
\hline $\begin{array}{l}\text { - } 2 \times \text { ET tubes } \\
\text { - Bougie/stylet } \\
\text { - LMA/Proseal/i-gel }\end{array}$ & & & & $\begin{array}{l}\checkmark \text { Intermediate area } \\
\text { - Doffing-protocol based } \\
\text { - Sanitize hands }\end{array}$ \\
\hline $\begin{array}{l}\text { - Closed suction } \\
\text { - Syringe/lube } \\
\text { - Nebulization port } \\
\text { - Closed suction } \\
\text { - Scalpel cricothyroidotomy kit } \\
\text { - Emergency drugs } \\
\text { - Sedation infusions }\end{array}$ & & & & $\begin{array}{l}\checkmark \text { Out of covid area } \\
\text { - Wipe-clean } \\
\text { - Respirator/footwear } \\
\text { - Sanitize hands } \\
\text { - Document intubation }\end{array}$ \\
\hline
\end{tabular}

Figure 2. Coronavirus disease 2019 (COVID-19) emergency intubation checklist. PPE: personal protective equipment; HME: heat and moisture exchanger; ET: endotracheal tube; LMA: laryngeal mask airway; RSI: rapid sequence intubation; BMV: bag mask ventilation; SGA: supraglottic airway device; FONA: front of neck access; BP: blood pressure; ECG: electrocardiogram; MACOCHA: MACOCHA intubation score; NG: nasogastric tube; NIV: noninvasive ventilation; HFNC: high-flow nasal cannula; IV: intravenous. Adapted with permission from Difficult Airway Society, UK [8].

bolism (PE) and decide on anticoagulation. This involved multidisciplinary teamwork, with the radiologists assisting by fasttracking the chest X-ray and CT reports. These test reports were based on pattern recognition and stated whether the findings were either suggestive or not suggestive of COVID-19, which helped us make decisions and to allocate the patients into suspect/confirmed COVID-19 areas.

Lung ultrasound has the advantages of reducing radiation, point-of-care use, and rapidity of evaluation. The examination is an adaptation of the Bedside lung ultrasound in emergency protocol designed by Lichtenstein [13]. On either side, features shown on three-point ultrasound scanning of the lung in COVID-19 include pleural thickening, nodularity, pleura/ lung interface shadows, B profile/C profile, and dynamic air bronchograms at the bases suggestive of consolidation changes. The ICS has suggested that if there is evidence of bi-basal consolidation, it will aid in a decision to prone the patient as shown in Figure 4B. Performing daily bedside focused intensive care echo, and lung ultrasound is advantageous as it can help recognise the above and the presence of right ventricular dysfunction/dilation, acute cor pulmonale, left ventricular dysfunction, and viral myopericarditis. Additionally, it can aid in assessing fluid responsiveness in these patients.

\section{Ventilator Management}

As mentioned earlier, ventilatory strategies differ based on the phenotypes. However, we utilised the pressure-volume loop analysis to understand a lower and an upper inflexion point. We performed PEEP titration using a combination of a recruitment manoeuvre followed by decremental PEEP. It is not uncommon to see these patients have low $\mathrm{PaO}_{2}$ and elevated $\mathrm{CO}_{2}$ caused by the shunt, which is more evident in patients with low lung compliance $\left(<40 \mathrm{~cm} \mathrm{H}_{2} \mathrm{O}\right)$. While inverse ratio ventilation and prone position ventilation are useful in patients with severe hypoxemia, airway pressure release ventilation (APRV) is a penultimate rescue strategy. Extracorporeal membrane oxygenation is a last resort option; it involves immediate referral to a specialised centre.

Decision making was guided based on a flowchart (Figure 5). Amato et al. [14] have mentioned possible mortality bene- 


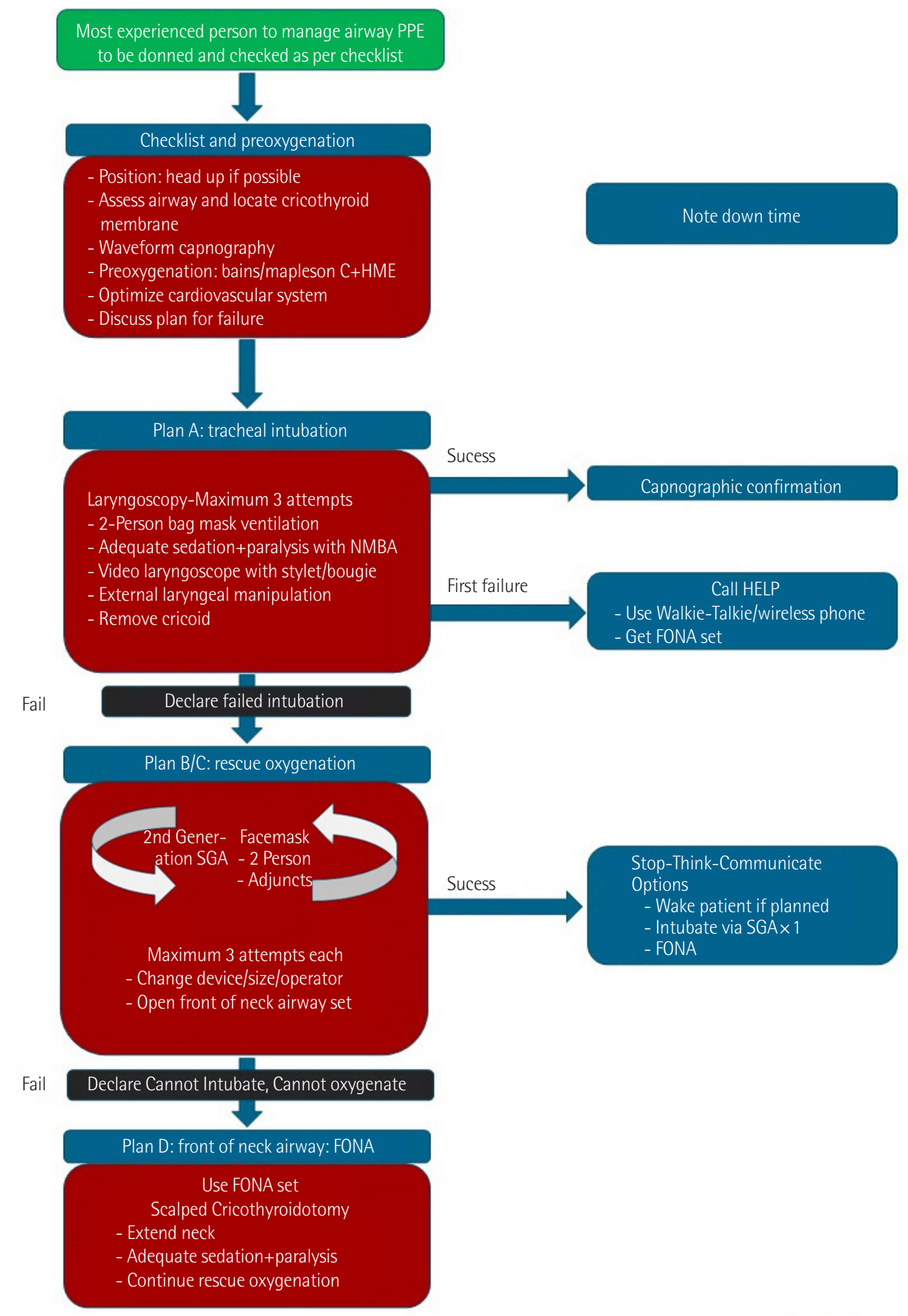

Figure 3. COVID-19 intubation pathway. PPE: personal protective equipment; HME: heat and moisture exchanger; NMBA: neuromuscular blocking agents; SGA: supraglottic airway; FONA: front of neck access. Adapted with permission from Difficult Airway Society, UK [8].

fits in ARDS patients with driving pressure-directed ventilator adjustments. However, the evidence is not substantial for a target driving pressure of less than $15 \mathrm{~cm} \mathrm{H}_{2} \mathrm{O}$, and its possi- bility of reducing mortality needs to be evaluated [15]. Prone position ventilation has shown a mortality benefit in ARDS, and early proning is a recommendation. It requires a team of 


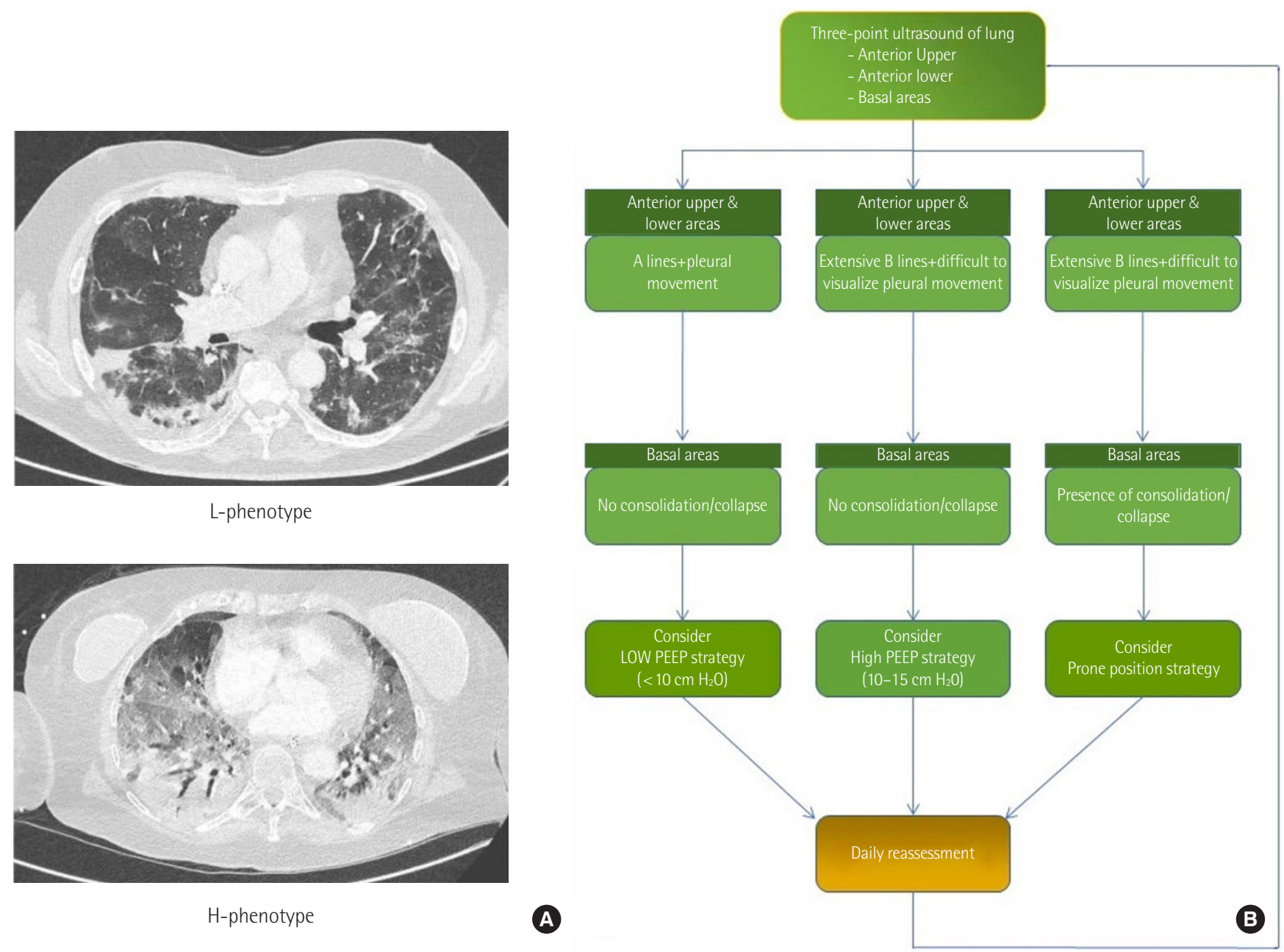

Figure 4. (A) High-resolution computed tomography of chest showing L and H phenotypes in coronavirus disease 2019 (COVID-19). (B) Lung ultrasound in COVID-19-decision-making flowchart. PEEP: positive end-expiratory pressure.

members trained in helping to turn the patient. Video aids and simulation training were incredibly useful, and we had a proning team that included operating department practitioners and theatre staff who helped us continuously. The preparation, timing, and advantages are mentioned in Table 1. We understand that time to initiate prone position ventilation may not be constant across all ICUs because of staffing and caseload issues. Still, essentially the aim is to provide 16 hours of prone position in 24 hours. At our unit proning is timed at 5 $\mathrm{PM}$ (unless required urgently, and patients with a $\mathrm{PaO}_{2} / \mathrm{FiO}_{2}$ $(\mathrm{P} / \mathrm{F})$ ratio of less than 150 underwent proning immediately after intubation. Problems associated with proning were facial oedema, a need for increased sedation/paralysis, and, most importantly, an inability to perform bedside echocardiography. A modification that helped us perform echocardiography in these patients included additional support/pillows at the chest level, which gave us access to the apical views, but this was difficult to achieve, as it was cumbersome given the nonconventional probe handling and positioning.

APRV is a relatively safe mode of ventilation, and patients on APRV have a higher P/F ratio increase than those on low tidal volume and synchronised intermittent ventilation [16]. However, as a nonconventional mode, there is a lag time for its acceptability. We used the APRV protocol in patients with poor compliance. The advantage of APRV was that the patient could breathe spontaneously. The weaning plan on APRV includes increasing the respiratory phase time (T high) and reducing the inspiratory pressure (P high).

Another issue worthy of mention is the securing of the junction points in the ventilator circuit. However, they reduced the chances of circuit disconnection and aerosol generation. We did observe the tapes slipping towards the lumen. Thus, we 


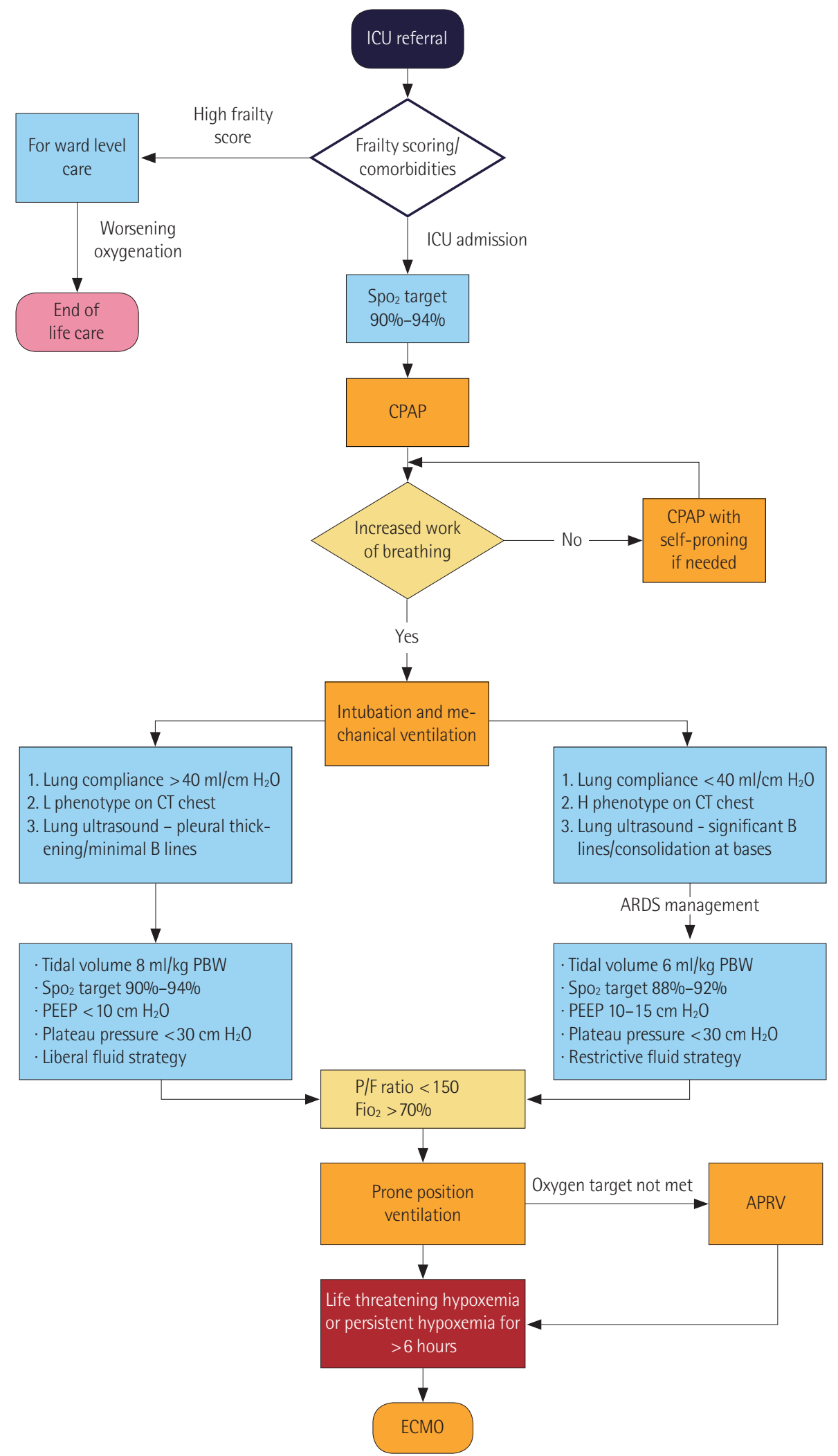

Figure 5. Approach to ventilatory management in coronavirus disease 2019 (COVID-19). ICU: intensive care unit; CPAP: continuous positive airway pressure; CT: computed tomography; PEEP: positive end-expiratory pressure; ARDS: acute respiratory distress syndrome; PBW: predicted body weight; $\mathrm{P} / \mathrm{F}: \mathrm{PaO}_{2} / \mathrm{FiO}_{2} ; \mathrm{APRV}$ : airway pressure release ventilation; ECMO: extracorporeal membrane oxygenation. 
had to be alert to this aspect since it was a preventable complication and should be avoided by serial examinations and monitoring. Any disconnection in a patient without spontaneous breathing required temporary clamping of the tube to reduce aerosol generation and avoid PEEP loss. In terms of heat and moisture exchanger/viral filters, we changed them every 24 hours.

\section{Tracheostomy}

The trend was such that, as time passed, we performed more tracheostomies, considering the need for long-term ventilation. Guided by the TracMan trial, we allowed liberal time before considering tracheostomy [17]. However, the trajectory was such that as we gained more understanding about the disease and the need for prolonged ventilation, there was a transition in decision making towards favouring tracheostomy in these patients. The vital step in reducing the aerosol-generating procedure was placing the ventilator on standby before using the dilator and resuming after connecting the ventilator circuit to the tracheostomy tube. We prefer performing bedside percutaneous tracheostomies with continuous bronchoscopy guidance. However, indications for surgical tracheostomy include the short neck, morbid obesity, and midline vessels' presence on a pre-procedural ultrasonographic scan of the neck.

\section{Hemodynamic Assessment and Fluid Responsiveness}

Initially, we followed a restrictive fluid balance approach, which changed with further literature reviews: the concept of better pulmonary perfusion with adequate fluid reserve is emerging, and therefore, unless patients progress into the H phenotype, a conservative fluid approach is suggested [18]. The passive

Table 1. Prone position ventilation in COVID-19: a team approach

\begin{tabular}{l} 
Prone position ventilation \\
\hline Preparation \\
Proning team training: using simulations/videos \\
Allocated theatre staff+anesthesiologist \\
Timing \\
Proning: 5 PM \\
Deproning: 9 AM \\
Advantage \\
Reduced workload on ICU staff \\
Lesser complications \\
Better allocation of human resources
\end{tabular}

COVID-19: coronavirus disease 2019; ICU: intensive care unit. leg raise test remains the most useful test given its simplicity and ease of reproducibility. It can lead to desaturation and non-availability of variables such as stroke volume and cardiac index that can produce difficulties with interpretation unless invasive cardiac output monitoring is available. Bedside echocardiography is useful to assess stroke volume variation, change in velocity-time integral, and distensibility index; however, it is not easy to obtain the required views, and the images may be suboptimal. While pulse pressure variation is a test available on most ICU monitors for patients receiving a tidal volume of $8 \mathrm{ml} / \mathrm{kg}$ with no spontaneous breaths, in patients on low tidal volume ventilation, a tidal volume challenge test appears to be a better option [19]. Furthermore, if there is the availability of invasive cardiac output monitoring, we believe the extravascular lung water index could provide additional information to guide fluid therapy.

\section{Pharmacological Therapy}

The details of this therapeutic component are beyond this article; however, we will summarise the present evidence. Dexamethasone in COVID-19 has gained interest due to better outcomes in multiple trials. The CoDEX trial evaluated the role of intravenous dexamethasone in moderate to severe ARDS related to COVID-19. The findings revealed a significantly increased number of ventilator-free days at day 28 than placebo [20]. Another multicentre study-REMAP-CAP trial included patients with severe COVID-19. The patients who received hydrocortisone showed superiority with organ support free days within day 21 [21]. The Recovery trial's preliminary results suggest reduced 28-day mortality in both patients on mechanical ventilatory support or those requiring oxygen therapy [22]. Finally, a metanalysis conducted in critically ill COVID-19 patients showed a lower 28-day all-cause mortality in patients treated with systemic steroids than placebo [23]. Our approach includes administering 6 milligrams of intravenous Dexamethasone for 10 days for all patients admitted to hospital requiring oxygen therapy.

Remdesivir has not been shown to result in a statistically significant reduction in mortality, but only reduces the duration of hospital stay [24]. Many patients have received it on a compassionate basis in a trial that demonstrated it has a potential role in patients with severe COVID-19 [25]. It is still under evaluation in multiple trials; however, no breakthrough evidence exists as of now. Prophylactic use of hydroxychloroquine has not prevented COVID-19 illness when administered within four days post-exposure [26]. Furthermore, its usefulness for the treatment of COVID-19 has not yet shown prom- 
ising results: a multicentre trial in non-severe COVID-19 patients showed no clinical improvement after 15 days of hydroxychloroquine. The groups included either hydroxychloroquine alone or in combination with azithromycin versus standard of care [27].

Similarly, drugs like lopinavir, ritonavir, favipiravir, and tocilizumab are under evaluation in multiple trials. The WHO Solidarity trial evaluated the role of remdesivir, hydroxychloroquine, lopinavir and interferon in patients hospitalized with COVID-19. The interim results showed no significant reduction in mortality, ventilatory support initiation, and hospital stay duration with the use of these medications [28]. Emerging data from REMAP-CAP trial in patients admitted to ICU with COVID-19 and treated with interleukin-6 (IL-6) inhibitors tocilizumab/sarilumab, have a trend towards reduced mortality and ventilator-free days when used in combination with steroids [29]. However, we are awaiting further analysis before using IL-6 inhibitors as the standard of care.

Although one publication mentions the benefit of convalescent plasma in COVID-19, the present evidence is lacking: an underpowered randomised trial on 103 patients did not show improvement in the clinical condition within 28 days [30]. Further studies focused on the possibility of cytokine storm as the plausible mechanisms of damage caused by the disease. Huet et al. [31] evaluated anakinra's role in severe cases and found that its administration is associated with reduced mortality and need for mechanical ventilation. Although this study had a low sample size, more extensive trials will help us understand its significance. Hyperbaric oxygen therapy was found to be of some benefit in a small sample size study [32]. However, large-scale studies are yet to evaluate the same, perhaps due to the availability of equipment and trained staff. We are awaiting the results of the Recovery, Recovery-RS, and REMAP-CAP trials, which will hopefully provide insight into more beneficial therapies.

Finally, with the availability of multiple vaccines and their fast-track approvals, large scale vaccination drives have been initiated in various countries. Although it is a significant development towards the fight against the pandemic, the longlasting socioeconomic deprivation will continue to impede the cessation of this dangerous infection. Hence, we believe we need to continue our efforts and manage COVID-19 with principles of best practice.

\section{Acute Kidney Injury}

Initial reports mentioned that COVID-19 did not cause acute kidney injury (AKI). With an increasing evidence base, it is clear that COVID-19 directly affects the kidneys, which is demonstrated by the presence of tubular damage on histopathological sections [33]. Most patients have low intravascular volume due to insensible loss, associated diarrhoea, and reduced intake. Therefore, an increasing number of patients could have pre-renal AKI or a combination of pre-renal and acute tubular necrosis, and the initial management would include fluid resuscitation. However, a fluid strategy needs regular review. There is a risk of causing fluid overload, in which case experts suggest using furosemide initially and then dialysis in cases of no response.

\section{Secondary Infections}

Secondary infections are common in COVID-19, especially in those who are on mechanical ventilation. We did not start antibiotics in newly admitted patients unless there were new chest X-ray/CT changes with a clinical pulmonary infection score greater than six suggestive of ventilator-associated pneumonia. It is not easy to differentiate between temperature spikes from COVID-19 and non-COVID-19 aetiology, and in such scenarios, we sent blood, urine, and sputum cultures for testing. Additional tests, such as beta-D glucan and galactomannan, are useful to aid in diagnosing fungal infections. There was some initial interest in using procalcitonin to evaluate secondary infections in COVID-19; however, National Institute for Health and Care Excellence recommendations are against this until further data is available for analysis. There can be elevated procalcitonin levels as the disease process increases in severity. An audit is underway at our centre to evaluate procalcitonin's role as an aid in antimicrobial stewardship.

\section{Deep Venous Thrombosis Prophylaxis}

These patients are usually in a prothrombotic state due to endothelitis, NETosis, hyperinflammation, and platelet dysfunction [34]. Thus, we designed a thromboprophylaxis pathway and administered high-dose anticoagulation (Figure 6). Nonetheless, the incidence of PE remained high. CTPA performed on these patients with persistent hypoxia will provide further evidence of whether there is a need to switch from a prophylactic dose to a treatment dose of low molecular weight heparin. Right ventricular dilation and impairment on bedside echocardiography offer additional clues about evidence of PE/acute cor pulmonale. Thus, daily bedside echocardiography leads to a focussed assessment and further helps with the decision making on dosing of anticoagulation. 


\section{Delirium}

A multitude of factors have made delirium an evident development; the occurrence of delirium is reported to be around $25 \%$ in initial studies and even higher in mechanically ventilated patients [35]. The incidence of delirium appears underreported, considering the increasing number of cases; therefore, it is an aspect of the condition that requires further mention. The multifactorial aetiology is shown in (Figure 7). In terms of management, additional factors need addressing: isolation, tendency to have fewer assessments, increased requirement for sedatives, prolonged ventilation, reduced mobility, and difficulty with communication. Our management was multidisciplinary, including routine examination, sedation holiday, low noise levels during night hours and curtailing the use of neuromuscular blocking agents (NMBA).

Courcelle et al. [36] conducted a multicentre observational trial that showed increased use of NMBA in COVID-19 compared to ARDS cases. Our approach includes the use of NMBA in the prone position and a daily review with a low threshold of stopping the infusions. The latter is performed based on the assessment of respiratory drive during spontaneous breaths. A high respiratory drive/effort will put the patient at risk of lung damage by patient self-inflicted lung injury. Hence, such patients need further continuation of NMBA. In awake patients, we organised video calls with family members. Other steps we focussed on were constant reassurance, counselling, moving the patient out of isolation areas once they were non-infective, and progressing to trips out of ICU areas, which improved their mental well-being.

\section{Prognosis}

An initial observational report from the European trial mentioned that critically ill patients with COVID-19 had 24\% mortality. The procalcitonin and IL-6 levels remained similar between survivors and non-survivors. Lower $\mathrm{P} / \mathrm{F}$ ratio, increased creatinine, D-Dimer, and ischemic heart disease are associated with increased ICU mortality [37]. A metanalysis did show variables contributing to poor prognosis in patients with $\mathrm{CO}$ VID-19. Patients aged $>70$ years had a 13-fold higher risk of dying compared to younger individuals. Males had higher odds

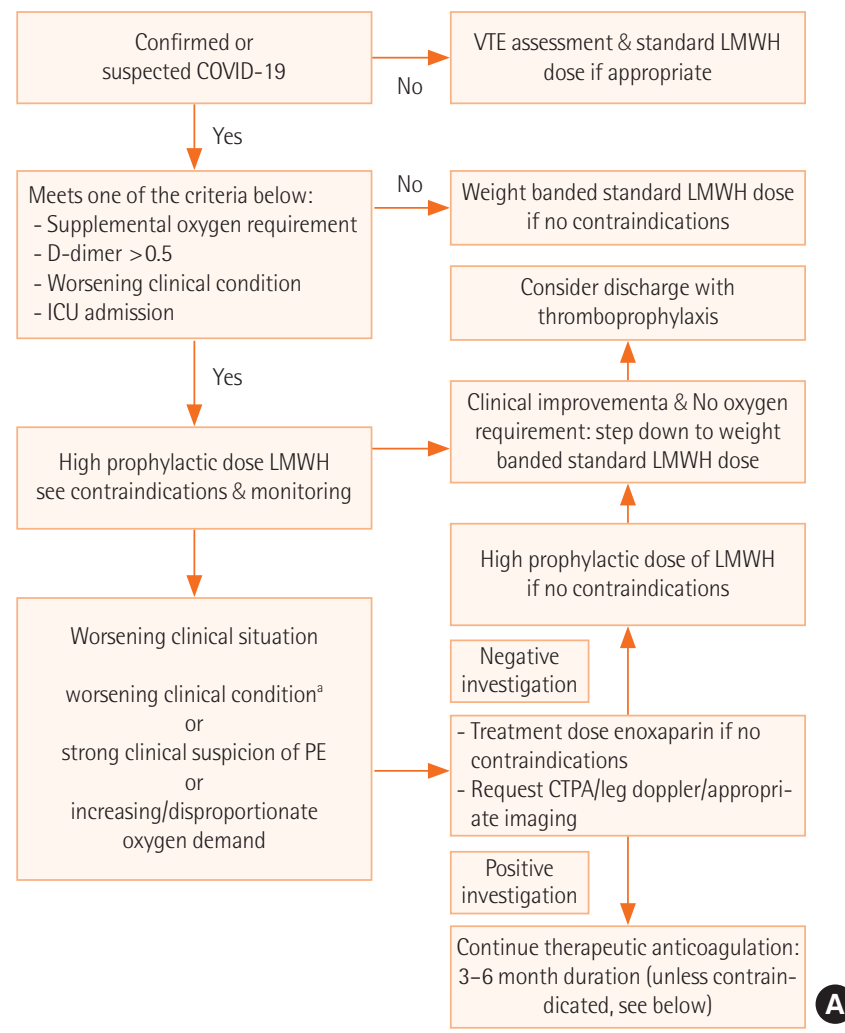

\begin{tabular}{|c|c|c|}
\hline \multicolumn{3}{|c|}{ Dalteparin dosing } \\
\hline Weight (kg) & $\begin{array}{l}\text { Prophylactic weight banded } \\
\text { standard dose (low-risk COV- } \\
\text { ID-19 patients including ESRF on } \\
\text { dialysis and } \mathrm{CrCl}<30 \mathrm{ml} / \mathrm{min} \text { ) }\end{array}$ & $\begin{array}{l}\text { High prophylactic dose } \\
\text { All ICU patients \& only in } \\
\text { patients with CrCl } \\
>30 \mathrm{ml} / \mathrm{min}\end{array}$ \\
\hline$<50$ & 2,500 units SC OD & 5,000 units SC OD \\
\hline $50-100$ & 5,000 units SC OD & 5,000 units SC BD \\
\hline $100-150$ & 5,000 units SC BD & 7,500 units SC BD \\
\hline$>150$ & 7,500 units SC BD & 10,000 units SC BD \\
\hline \multicolumn{3}{|c|}{$\begin{array}{l}\text { LMWH high prophylactic doses in severe renal impairment } \\
\qquad(\mathrm{CrCl}<30 \mathrm{ml} / \mathrm{min} \text { or on dialysis }) \\
\text { Monitor anti-Xa levels }\end{array}$} \\
\hline Weight (Kg) & \multicolumn{2}{|c|}{$\begin{array}{c}\text { High prophylactic dose } \\
\text { Enoxaparin }\end{array}$} \\
\hline$<50$ & \multicolumn{2}{|c|}{$40 \mathrm{mg} \mathrm{SC} \mathrm{OD}$} \\
\hline $50-100$ & \multicolumn{2}{|c|}{$50 \mathrm{mg} \mathrm{SC} \mathrm{OD}$} \\
\hline $100-125$ & \multicolumn{2}{|c|}{$80 \mathrm{mg} \mathrm{SC} \mathrm{OD}$} \\
\hline$>125$ & \multicolumn{2}{|c|}{$50 \mathrm{mg} \mathrm{SC} \mathrm{BD}$} \\
\hline \multicolumn{3}{|c|}{ Enoxaparin treatment dose } \\
\hline $\begin{array}{l}\mathrm{CrCl} \text { using Cockcroft-Gault } \\
\text { equation }\end{array}$ & Treatment dose & Monitoring \\
\hline$>30 \mathrm{ml} / \mathrm{min}$ & $1 \mathrm{mg} / \mathrm{kg} \mathrm{BD}$ & $\mathrm{FBC}, \mathrm{U}+\mathrm{E}$, clotting \\
\hline$<30 \mathrm{ml} / \mathrm{min}$ or dialysis & $1 \mathrm{mg} / \mathrm{kg}$ OD & $\begin{array}{l}\mathrm{FBC}, \mathrm{U}+\mathrm{E} \text {, clotting \& } \\
\text { anti-Xa monitoring }\end{array}$ \\
\hline \multicolumn{3}{|c|}{$\begin{array}{l}\text { Cockcroft-Gault } \mathrm{CrCl}, \mathrm{mL} / \mathrm{min}=(140 \text {-age }) \times(\text { adjusted body weight, } \mathrm{kg}) \times(1.23 \text { if male, } \\
1.04 \text { if female }) /(\mathrm{Crumol} / /) \\
\text { Adjusted body weight, } \mathrm{kg}=\mathrm{IBW}, \mathrm{kg}+0.4 \times(\text { actual body weight, } \mathrm{kg}-\mathrm{IBW}, \mathrm{kg})\end{array}$} \\
\hline
\end{tabular}

Figure 6. (A) Thromboprophylaxis pathway in coronavirus disease 2019 (COVID-19). (B) Low molecular weight heparin dosing in COVID-19. VTE: venous thromboembolism; LMWH: Iow molecular weight heparin; ICU: intensive care unit; PE: pulmonary embolism; CTPA: computed tomography pulmonary angiography; NEWS: National Early Warning Score; ESRF: end stage renal failure; CrCl: creatinine clearance; SC: subcutaneous; OD: once a day; BD: twice a day; FBC: full blood count; U+E: urea/creatinine and electrolytes; IBW: ideal body weight. ${ }^{a} \mathrm{Clini}$ cal improvement/deterioration refers to changes in NEWS, blood test results, and/or radiology. 


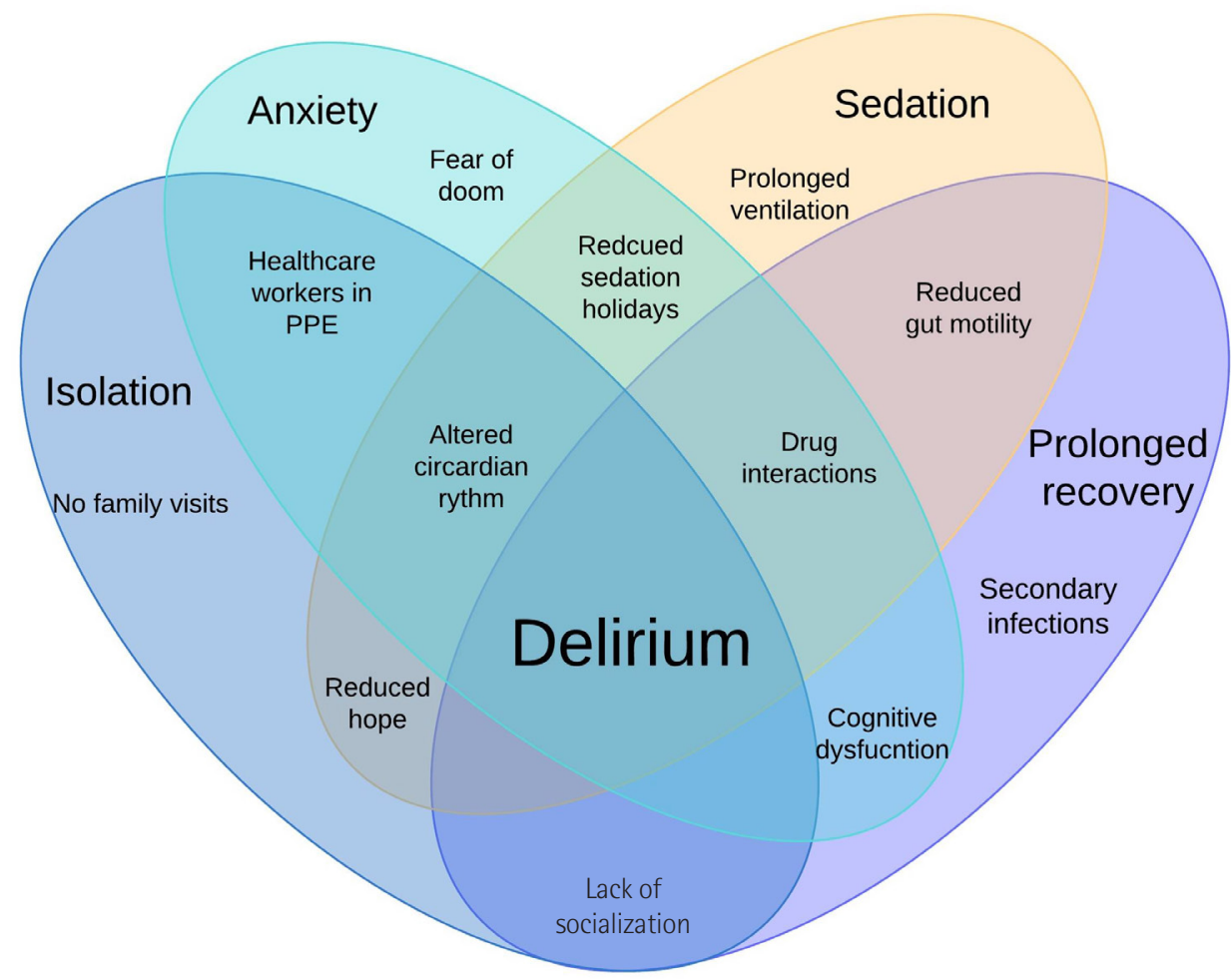

Figure 7. Factors contributing to delirium in coronavirus disease 2019 (COVID-19).

of dying than females. Amongst those with AKI, liver injury, and acute cardiac injury, the patients with the latter had the worst outcomes [38]. Liang et al. [39] have mentioned COVIDGRAM - a calculator that predicts the progression to severe disease in patients. This tool is validated, although further studies are needed to understand its predictability.

\section{Socioemotional Factors and Burnout}

PPE has been ubiquitous in the present scenario, and caring for patients while wearing this newfound armour has not been comfortable for all. Dehydration and anxiety were a few of the many associated adverse effects evident in staff members. Donning and doffing the PPE, which is a critical step, also demanded significant time and energy. The shields and masks hampered communication between staff and patients; the respirator/mask led to hampered communication between staff members. We used simple walkie-talkie devices to communicate with teams in the isolation areas. These proved helpful even in cases of emergencies, where sign language can be less informative. Managing working hours had a significant impact on staff well-being. The nursing staff received regular two-hourly breaks, coordinated by a senior team member. We agreed that this led to increased PPE use, but this could be balanced against a more positive mindset while working in such a stressful environment. Each of our isolation bays consisted of six patients, based on the ICU areas that were allocated to each doctors' respective teams. The patients' initial daily assessment included reviewing electronic data, discussing patient progress with the nursing team over the walkie talkie, and documenting a draft plan for each patient. A consultant and another doctor would enter the COVID-19 areas with full PPE to assess the patient and finalise their plans. In the case of an emergency or new admission, an airway-trained doctor would escort the patient from the ED or ward and then perform the necessary procedures. At the same time, the other doctor would remain outside the COVID-19 bay and help with the documentation. We suggest using electronic prescribing, which reduces extra visits into the COVID-19 areas and leads to the appropriate use of PPE.

The above steps significantly reduced mental fatigue and helped us deliver better patient care. Regarding communication with families, this aspect has been more difficult than others. As a result of the lockdown, the families could not personally visit the hospital because of the high risk of COVID-19 transmission; therefore, we had to adapt to non-conventional modes of communication. While the awake patients spoke to their families using video calls, the family members of those on ventilators had daily telephone discussions with the doc- 
tors, which helped them cope with their stress and anxiety. Consent for procedures was also via audio or video calls, and the associated complications were explained. Breaking bad news over the phone led to emotional turmoil in both the family and the doctors/nurses involved in the discussion. However, as the lockdown rules eased in a graded manner, families of the patients requiring end-of-life care could visit our unit and were given PPE after performing a mask fit test. Dealing with ethical issues has been in line with recommendations from the ICS. The discussions about medical decisions and the consent of family members in digital format are indeed complex. Using tablets, phones, and digital media to obtain signatures on consent and discussion forms seems to be a plausible option, provided the electronic documents are accessed and stored securely.

\section{CONCLUSIONS}

As more evidence evolves about the disease process of COVID-19, the treatment modalities used need to undergo dynamic changes. Due to multiple resurgences globally and the associated socio-economic impact, infrastructure and advanced planning components should be at the forefront. Protocols and policies help to triage patients for admission to the ICU. Multidisciplinary involvement including- Intensivists, pulmonologists, anaesthesiologists, radiologists, infectious disease specialists, the paramedical and non-medical staff is paramount

For those patients progressing to mechanical ventilation, low tidal volume ventilation and early proning are of utmost importance. Monitoring pulmonary parenchymal function is aided with bedside ultrasonography; this is an alternative to HRCT and avoids risky transfers, the occurrence of desaturation, and increased spreading of aerosols. Bedside ultrasound has further benefit of detecting features of acute cor pulmonale or $\mathrm{PE}$, which is vital given the associated prothrombotic state of COVID-19. The initial fluid balance approach should aim for euvolaemia, as patients are intravascularly volume depleted. In contrast, a restrictive policy is better for those with features of ARDS. As for pharmacological treatment, steroids are the only class of drugs to have shown mortality benefit in trials. All the other groups of medicines have either been found to be not significantly beneficial or are still under investigation.

Mortality has a direct relationship to increasing age; it is also higher in those with ischaemic heart disease and acute cardiac injury. Other factors that suggest poor prognosis include a lower $\mathrm{P} / \mathrm{F}$ ratio, AKI occurrence, and thrombocyto- paenia. The socio-emotional component needs special mention, encompassing burnout on the medical team and stress on patients and their family members. Adapting to newer communication methods is useful in overcoming barriers due to PPE and isolation and is also helpful in reducing the occurrence of delirium.

\section{CONFLICT OF INTEREST}

No potential conflict of interest relevant to this article was reported.

\section{ORCID}

Avinash Kumar Jha https://orcid.org/0000-0002-0474-5953 Sudhindra Gurunath Kulkarni

https://orcid.org/0000-0001-6914-4473

\section{AUTHOR CONTRIBUTIONS}

Conceptualisation: all authors. Data curation: AKJ. Formal analysis \& Methodology: all authors. Project administration: SGK. Visualisation \& Writing-original draft: AKJ. Writing-review \& editing: all authors.

\section{REFERENCES}

1. Bamford P, Bentley A, Dean J, Whitmore D, Wilson-Baig N. ICS Guidance for Prone Positioning of the Conscious COVID-19 Patient 2020 [Internet]. London: Intensive Care Society; 2020 [cited 2021 Feb 19]. Available from: https://www.ics. ac.uk/ICS/Pdfs/COVID-19/Guidance_for_conscious_proning.aspx.

2. Caputo ND, Strayer RJ, Levitan R. Early self-proning in awake, non-intubated patients in the emergency department: a single ed's experience during the COVID-19 pandemic. Acad Emerg Med 2020;27:375-8.

3. Gattinoni L, Chiumello D, Caironi P, Busana M, Romitti F, Brazzi L, et al. COVID-19 pneumonia: different respiratory treatments for different phenotypes? Intensive Care Med 2020; 46:1099-102.

4. Eddleston J, Pittard A. Letter regarding the use of continuous positive airway pressure (CPAP) for COVID-19 positive patients [Internet]. London: Faculty of Intensive Care Medicine; 2020 [cited 2021 Feb 19]. Available from: https://www.ficm. ac.uk/news-events-education/news/letter-regarding-use-continuous-positive-airway-pressure-cpap-COVID-19. 
5. Zhou F, Yu T, Du R, Fan G, Liu Y, Liu Z, et al. Clinical course and risk factors for mortality of adult inpatients with COVID-19 in Wuhan, China: a retrospective cohort study. Lancet 2020;395:1054-62.

6. Barrasa H, Rello J, Tejada S, Martin A, Balziskueta G, Vinuesa C, et al. SARS-CoV-2 in Spanish Intensive Care Units: Early experience with 15-day survival in Vitoria. Anaesth Crit Care Pain Med 2020;39:553-61.

7. Australian and New Zealand Intensive Care Society. The Australian and New Zealand Intensive Care Society (ANZICS) COVID-19 guidelines [Internet]. Camberwell: Australian and New Zealand Intensive Care Society; 2020 [cited 2021 Feb 19]. Available from: http://cec.health.nsw.gov.au/_data/assets/ pdf_file/0004/572512/ANZICS-COVID-19-Guidelines-Version-1.pdf.

8. Difficult Airway Society. DAS ICU intubation guidelines [Internet]. London: Difficult Airway Society; 2017 [cited 2021 Jan 25]. Available from: https://das.uk.com/files/2017/page/DAS_ ICU_guidelines.pdf.

9. Rello J, Storti E, Belliato M, Serrano R. Clinical phenotypes of SARS-CoV-2: implications for clinicians and researchers. Eur Respir J 2020;55:2001028.

10. Ferrando C, Suarez-Sipmann F, Mellado-Artigas R, Hernán$\operatorname{dez}$ M, Gea A, Arruti E, et al. Clinical features, ventilatory management, and outcome of ARDS caused by COVID-19 are similar to other causes of ARDS. Intensive Care Med 2020;46:220011.

11. Calfee CS, Delucchi K, Parsons PE, Thompson T, Ware L, Matthay M, et al. Subphenotypes in acute respiratory distress syndrome: latent class analysis of data from two randomised controlled trials. Lancet Respir Med 2014;2:611-20.

12. Ai T, Yang Z, Hou H, Zhan C, Chen C, Lv W, et al. Correlation of chest CT and RT-PCR testing for coronavirus disease 2019 (COVID-19) in China: a report of 1014 cases. Radiology 2020; 296:E32-40

13. Lichtenstein D. Lung ultrasound in the critically ill. Curr Opin Crit Care 2014;20:315-22.

14. Amato MB, Meade MO, Slutsky AS, Brochard L, Costa EL, Schoenfeld DA, et al. Driving pressure and survival in the acute respiratory distress syndrome. N Engl J Med 2015;372:747-55.

15. Bugedo G, Retamal J, Bruhn A. Driving pressure: a marker of severity, a safety limit, or a goal for mechanical ventilation? Crit Care 2017;21:199.

16. Sun X, Liu Y, Li N, et al. The safety and efficacy of airway pressure release ventilation in acute respiratory distress syndrome patients: a PRISMA-compliant systematic review and metaanalysis. Medicine (Baltimore) 2020;99:e18586.
17. Young D, Harrison DA, Cuthbertson BH, Rowan K, TracMan Collaborators. Effect of early vs late tracheostomy placement on survival in patients receiving mechanical ventilation: the TracMan randomized trial. JAMA 2013;309:2121-9.

18. Robba C, Battaglini D, Ball L, Patroniti N, Loconte M, Brunetti $\mathrm{L}$, et al. Distinct phenotypes require distinct respiratory management strategies in severe COVID-19. Respir Physiol Neurobiol 2020;279:103455.

19. Myatra SN, Monnet X, Teboul JL. Use of 'tidal volume challenge' to improve the reliability of pulse pressure variation. Crit Care 2017;21:60.

20. Tomazini BM, Maia IS, Cavalcanti AB, Berwanger O, Rosa $\mathrm{R}$, Veiga $\mathrm{V}$, et al. Effect of dexamethasone on days alive and ventilator-free in patients with moderate or severe acute respiratory distress syndrome and COVID-19: the CoDEX randomized clinical trial. JAMA 2020;324:1307-16.

21. Angus DC, Derde L, Al-Beidh F, Annane D, Arabi Y, Beane A, et al. Effect of hydrocortisone on mortality and organ support in patients with severe COVID-19: the REMAP-CAP COVID-19 corticosteroid domain randomized clinical trial. JAMA 2020; 324:1317-29.

22. RECOVERY Collaborative Group, Horby P, Lim WS, Emberson JR, Mafham M, Bell LJ, et al. Dexamethasone in hospitalized patients with covid-19: preliminary report. N Engl J Med. 2020 Jul 17 [Epub]. https://doi.org/10.1056/NEJMoa2021436.

23. WHO Rapid Evidence Appraisal for COVID-19 Therapies (REACT) Working Group, Sterne JA, Murthy S, Diaz J, Slutsky A, Villar J, et al. Association between administration of systemic corticosteroids and mortality among critically Ill patients with COVID-19: a meta-analysis. JAMA 2020;324:133041.

24. Wang Y, Zhang D, Du G, Du R, Zhao J, Jin Y, et al. Remdesivir in adults with severe COVID-19: a randomised, double-blind, placebo-controlled, multicentre trial. Lancet 2020;395:156978.

25. Grein J, Ohmagari N, Shin D, Diaz G, Asperges E, Castagna A, et al. Compassionate use of Remdesivir for patients with severe covid-19. N Engl J Med 2020;382:2327-36.

26. Boulware DR, Pullen MF, Bangdiwala AS, Pastick KA, Lofgren SM, Okafor EC, et al. A randomized trial of hydroxychloroquine as postexposure prophylaxis for covid-19. N Engl J Med 2020;383:517-25.

27. Cavalcanti AB, Zampieri FG, Rosa RG, Azevedo LC, Viega VC, Avezum A, et al. Hydroxychloroquine with or without Azithromycin in Mild-to-Moderate Covid-19. N Engl J Med 2020;383: 2041-52.

28. WHO Solidarity Trial Consortium, Pan H, Peto R, Henao-Re- 
strepo AM, Preziosi MP, Sathiyamoorthy V, et al. Repurposed antiviral drugs for covid-19: interim WHO solidarity trial results. N Engl J Med 2021;384:497-511.

29. Interim position statement: interleukin-6 inhibitors (tocilizumab or sarilumab) for patients admitted to ICU with COVID-19 pneumonia (adults) [Internet]. Redditch: NHS England; 2021 [cited 2021 Jan 20]. Available from: https://www. cas.mhra.gov.uk/ViewAndAcknowledgment/viewAttachment.aspx?Attachment_id=103715.

30. Li L, Zhang W, Hu Y, Tong X, Zheng S, Yang J, et al. Effect of convalescent plasma therapy on time to clinical improvement in patients with severe and life-threatening COVID-19: a randomized clinical trial. JAMA 2020;324:460-70.

31. Huet T, Beaussier H, Voisin O, Jouveshomme S, Dauriat G, Lazareth I, et al. Anakinra for severe forms of COVID-19: a cohort study. Lancet Rheumatol 2020;2:e393-400.

32. Thibodeaux K, Speyrer M, Raza A, Yaakov R, Serena T. Hyperbaric oxygen therapy in preventing mechanical ventilation in COVID-19 patients: a retrospective case series. J Wound Care 2020;29:S4-8.

33. Su H, Yang M, Wan C, Yi L, Tang F, Zhu H, et al. Renal histopathological analysis of 26 postmortem findings of patients with COVID-19 in China. Kidney Int 2020;98:219-27.
34. Becker RC. COVID-19 update: covid-19-associated coagulopathy. J Thromb Thrombolysis 2020;50:54-67.

35. Kotfis K, Williams Roberson S, Wilson JE, Dabrowski W, Pun BT, Ely EW. COVID-19: ICU delirium management during SARS-CoV-2 pandemic. Crit Care 2020;24:176.

36. Courcelle R, Gaudry S, Serck N, Blonz G, Lascarrou JB, Grimaldi D, et al. Neuromuscular blocking agents (NMBA) for COVID-19 acute respiratory distress syndrome: a multicenter observational study. Crit Care 2020;24:446.

37. Wendel Garcia PD, Fumeaux T, Guerci P, Heuberger DM, Montomoli J, Roche-Campo F, et al. Prognostic factors associated with mortality risk and disease progression in 639 critically ill patients with COVID-19 in Europe: Initial report of the international RISC-19-ICU prospective observational cohort. EClinicalMedicine 2020;25:100449.

38. Figliozzi S, Masci PG, Ahmadi N, Tondi L, Koutli E, Aimo A, et al. Predictors of adverse prognosis in COVID-19: a systematic review and meta-analysis. Eur J Clin Invest 2020;50:e13362.

39. Liang W, Liang H, Ou L, Chen B, Chen A, Li C, et al. Development and validation of a clinical risk score to predict the occurrence of critical illness in hospitalized patients with COVID-19. JAMA Intern Med 2020;180:1081-9. 